\title{
Triptonide Modulates MAPK Signaling Pathways and Exerts Anticancer Effects via ER Stress-Mediated Apoptosis Induction in Human Osteosarcoma Cells
}

This article was published in the following Dove Press journal:

Cancer Management and Research

\author{
Liyun Zheng ${ }^{1,2, *}$ \\ Shiji Fang ${ }^{1,2, *}$ \\ Junguo Hui ${ }^{3}$ \\ Vinothkumar Rajamanickam² \\ Minjiang Chen ${ }^{2}$ \\ Qiaoyou Weng (iD ${ }^{2}$ \\ Xulu $\mathrm{Wu}^{2}$ \\ Zhongwei Zhao' \\ Jiansong Ji (iD) ${ }^{1-3}$ \\ 'Interventional Diagnosis and Treatment \\ Center, Lishui Hospital of Zhejiang \\ University, Lishui, Zhejiang 323000, \\ People's Republic of China; ${ }^{2}$ Key \\ Laboratory of Imaging Diagnosis and \\ Minimally Invasive Intervention Research, \\ Lishui Hospital of Zhejiang University, \\ Lishui, Zhejiang 323000, People's \\ Republic of China; ${ }^{3}$ Department of \\ Radiology, Lishui Hospital of Zhejiang \\ University, Lishui, Zhejiang 323000, \\ People's Republic of China
}

*These authors contributed equally to this work
Correspondence: Jiansong Ji Lishui Hospital of Zhejiang University, Lishui, Zhejiang 323000, People's Republic of China

Tel +86-13648782878

Fax +86-578-2133457

Email Ischrjjs@I63.com
Background: Osteosarcoma (OS) is the most common primary malignancy arise from bone and is one of the causes of cancer-related deaths. Triptonide (TN), a diterpenoid epoxide presented in Tripterygium wilfordii, is shown to possess a broad spectrum of biological properties.

Methods: In this study, we investigate the growth inhibitory effect of TN against human OS cells and its underlying molecular mechanism of action.

Results: Findings of our in vitro study revealed that TN exhibited a dose-dependent cytotoxic effect in MG63 and U-2OS cells. ROS-mediated cytotoxic effect was achieved in OS cells treated with TN which was reversed upon NAC treatment. Significantly, increased expression of PERK, p-EIF2, GRP78, ATF4 and CHOP in TN-treated OS cells unfolds the molecular mechanism of TN targets ER stress-mediated apoptosis. Modulation of ERK MAPK pathway was also observed as evidenced by the increased phosphorylation of ERK (p-ERK) and p-p38 in TN-treated OS cells.

Conclusion: Altogether, the outcome of the study for the first time revealed that TN exhibited its potential chemotherapeutic effects through ROS-mediated ER stress-induced apoptosis via p38 and ERK MAPK signaling pathways.

Keywords: osteosarcoma, triptonide, ER stress, apoptosis, MAPK signaling

\section{Introduction}

Osteosarcoma (OS) is the most common primary malignancy arise from bone, with an incidence rate of 10 to 26 per million new cases worldwide each year. ${ }^{1}$ As a high-grade malignant tumor frequently found in children and adolescents, OS is treated by various therapeutic methods ranging from surgery alone, surgery combined with (cisplatin, methotrexate, cyclophosphamide and adriamycin, etc.) and radiation with 5-year survival rate being $60-70 \% .^{2}$ However, there were no significant improvements in the long-term survival of a group of patients who are resistant to chemotherapy, prone to local recurrence or distant metastasis. ${ }^{3}$ Hence, development of sensitive and novel therapeutics from natural sources is always in demand to overcome the shortfalls of current cancer therapeutics. ${ }^{4}$

The whole plant of Tripterygium wilfordii has long been used in traditional Chinese medicine for the treatment of autoimmune disorders such as rheumatoid arthritis, systemic lupus and ankylosing spondylitis. ${ }^{5,6}$ But still Tripterygium 
wilfordii has limited clinical application due to its severe toxicity toward vital organs including liver. ${ }^{7}$ However, active ingredients such as triptolide and celastrol extracted from tripterygium had shown to possess anti-inflammatory and anti-tumor effects both in in vivo and in vitro. ${ }^{8,9}$ Anticancer effect of triptonide (TN), the other diterpene epoxide constituent of Tripterygium wilfordii, was demonstrated in pancreatic, ${ }^{10}$ nasopharyngeal, lung ${ }^{11}$ and lymphoma cancer. $^{12}$ However, until recently, its growth inhibitory effect on osteosarcoma and its underlying molecular mechanism have not uncovered in detail.

The imbalance of anti-oxidation and pro-oxidation in tumor cell cause the elevated endogenous reactive oxygen species (ROS). The elevated ROS facilitates cell progression such as proliferation, migration and drug resistance in tumor cells. ${ }^{13}$ However, the ROS is a "double-edged sword", the excess content of ROS may result in growth inhibition and apoptosis. ${ }^{14-16}$ ROS acts as crucial regulators of endoplasmic reticulum stress (ER) stress, which induces unfolded protein response (UPR) to prevent tumor cells from death. ${ }^{17}$ During the UPR, the chaperone proteins, such as glucose-regulated protein 78 (GRP78) that binds to three trans-membrane proteins like inositol requiring enzyme 1 (IRE1), PKR-like ER kinase (PERK) or activating transcription factor 6 (ATF 6). ${ }^{13}$ The sustained ER stress without protective effect of UPR eventually results in apoptosis with up-regulation of characteristically pro-apoptotic proteins including C/EBP homologous transcription factor protein (CHOP) and ER-associated caspase $12 .^{13}$ Studies had demonstrated that targeting the oxidative stress-mediated ER stress is one of the effective strategies for tumor therapy. ${ }^{18-20}$ Yang et $\mathrm{al}^{18}$ found that $\omega$ Hydroxyundec-9-enoic acid induced apoptosis through ROS-mediated endoplasmic reticulum stress in non-small cell lung cancer cells. Wu et $\mathrm{al}^{19}$ indicated that bicyclol exerted an anti-tumor effect via ROS-mediated ER stress in human renal cell carcinoma cells. Rajamanickam et $\mathrm{al}^{20}$ suggested that allylated curcumin analog CA6 inhibited proliferation of gastric cancer cells though ROS-ER stress axis. However, the effect of $\mathrm{TN}$ on the ROS-ER stress axis remained unclear. We aimed to identify the mechanisms underlying the inhibitory effects of $\mathrm{TN}$ in $\mathrm{OS}$ cells. We show that TN inhibited the growth of OS cells through ROSdependent activation of the ER stress and induction of apoptosis. Importantly, our results show that $\mathrm{TN}$ treatment increased the phosphorylation of p38 and ERK MAPK but not JNK MAPK. The activation of p38 and ERK MAPK mediated the ER stress in OS to induce cytotoxicity. We for the first time reported the anti-tumor potential of TN along with molecular mechanism in TN-treated OS cells in vitro.

\section{Materials and Methods Cell Culture}

The human osteosarcoma cell lines MG63 and U-2OS were obtained from the Chinese Academy of Sciences Cell Bank (Shanghai, China). U-2OS cells were grown in high-glucose RPMI-1640 medium (Gibco, Grand Island, NY, USA) whereas MG63 cells were grown in high-glucose $\alpha$-MEM medium, supplemented with 10\% fetal bovine serum (Gibco, Grand Island, NY, USA) and antibiotic solution $(100 \mu \mathrm{g} / \mathrm{mL}$ penicillin and $100 \mu \mathrm{g} / \mathrm{mL}$ streptomycin, Sigma, St. Louis, $\mathrm{MO}, \mathrm{USA})$. Both the cell lines were maintained in an incubator at $37^{\circ} \mathrm{C}$ with $5 \% \mathrm{CO}_{2}$.

\section{Chemicals and Antibodies}

Triptonide (purity $>98 \%$ ) was purchased from Sigma Chemicals Co. (St. Louis, MO, USA) and dissolved in dimethyl sulfoxide (DMSO) to obtain different stock concentrations and then diluted in the culture medium at various concentrations for different experiments. 3-[4, 5-dimethylthylthia-zol-2-yl]-2, 5 diphenyltetrazolium bromide (MTT) and N-acetyl-L-cysteine (NAC) were also obtained from Sigma Chemicals Co. (St. Louis, MO, USA). Antibodies against p38 MAPK (\#9212), Phosphop38 MAPK (\#9215), JNK (\#9252), Phospho-JNK (\#4671), Phospho-p44/42 MAPK (Erk1/2) (\#9101), p44/42 MAPK (Erk1/2) (\#9102), $\beta$-Actin (\#3700), Cleaved Caspase-3 (\#9664), Bcl-2 (\#15,071), Bcl-xL (\#2764), PERK (\#3192), Phospho-eIF2 $\alpha$ (\#3597), GRP78 (\#3183), CHOP (\#2895) and HRP-linked secondary antibodies Anti-rabbit IgG (\#7074) and Anti-mouse IgG (\#7076) were purchased from Cell Signaling Technology (Shanghai, China). Antibody AFF4 (ab103586) was purchased from Abcam (Shanghai, China). The study was conducted in accordance with the Basic \& Clinical Pharmacology \& Toxicology policy for experimental and clinical studies. ${ }^{21}$

\section{Cell Growth Assay}

The cell viability was measured by the 3-[4,5-dimethylthylthiazol-2-yl]-2,5 diphenyltetrazolium bromide (MTT) assay. Briefly, cells were seeded in 96well plates at a density of $2 \times 10^{5}$ cells $/ \mathrm{cm}^{2}$. After the indicated treatment for $48 \mathrm{~h}, 20 \mu \mathrm{L}$ MTT solution $(5 \mathrm{mg} /$ $\mathrm{mL}$ ) was added to each well, cells were further incubated in $\mathrm{CO}_{2}$ incubator for $4 \mathrm{~h}$ at $37^{\circ} \mathrm{C}$. Afterwards, DMSO 
$(150 \mathrm{~mL} /$ well $)$ was added to dissolve formazan crystals. The optical density (OD) of each well was observed at a wavelength of $490 \mathrm{~nm}$ using a microplate spectrophotometer (Molecular Devices, Sunnyvale, CA, USA). Cell viability was calculated according to the following formula: cell viability $=(\mathrm{OD}$ treatment $/ \mathrm{OD}$ control $) \times$ $100 \%$. The $\mathrm{IC}_{50}$, defined as the drug concentration at which cell growth was inhibited by $50 \%$.

\section{ROS Production Assay}

The intracellular ROS production was determined by Reactive Oxygen Species Assay Kit (Beyotime Biotechnology, Shanghai, China). Briefly, cells were seeded in a 6-well plate for $24 \mathrm{~h}$ after treatment with TN for the next $48 \mathrm{~h}$, the cells were harvested and suspended in PBS buffer. The cell suspensions were added with medium containing 2',7'-dichlorodihydrofluorescein diacetate (H2DCF-DA) dye and then incubated for $30 \mathrm{~min}$ at $37^{\circ}$ C. The fluorescent intensities were quantified using a flow cytometry. Cellular ROS level in the treatment group was normalized to that of the control group.

\section{Apoptosis Assay}

Cell apoptosis was detected by the Annexin V/Propidium iodide (PI) Apoptosis detection kit (Biyuntian, Shanghai, China) according to the manufacturer's protocol. Briefly, cells were grown in 6-well plate and then treated with indicated dosage of $\mathrm{TN}$ for $48 \mathrm{~h}$ and collected. Cells were resuspended with $196 \mu \mathrm{L}$ binding buffer containing $5 \mu \mathrm{L}$ Annexin V and $10 \mu \mathrm{L}$ PI fluorescence dye and then apoptosis was detected by flow cytometry (Becton Dickinson FACS Calibur).

\section{Western Blot Analysis}

Cells were collected by scraping in whole-cell lysis buffer. Lysates were mixed and incubated on ice for $10 \mathrm{~min}$, and then cell debris was spun down at a speed of $10,000 \mathrm{~g}$ for 10 min. The protein concentrations were measured by the Bradford protein assay kit (Bio-Rad, Hercules, CA). Aliquots of $40 \mu \mathrm{g}$ protein from each sample were separated by $10 \%$ SDS-polyacrylamide gel electrophoresis (SDSPAGE) and transferred onto a polyvinylidene difluoride (PVDF) membrane (Millipore, Bedford, MA). Membranes were blocked with $10 \%$ skimmed milk for $1 \mathrm{~h}$ and then incubated with specific primary antibody (1:1000) overnight at $4^{\circ} \mathrm{C}$, followed by incubation with corresponding secondary antibody (HRP-conjugated anti-rabbit or anti-mouse IgG at 1:3000) for $30 \mathrm{~min}$ to $1 \mathrm{~h}$ at room temperature. Antibody binding was visualized by enhanced chemiluminescence (ECL) detection system (Amersham Biosciences, Piscataway, NJ). The intensity of interested band was quantified using Image $\mathbf{J}$ software, and the value was normalized to corresponding loading controls.

\section{siRNA Transfection}

Briefly, MG63 cells were seeded in a six well plate at a density of $2 \times 10^{5}$ for $24 \mathrm{~h}$. Then, cells were transfected with siRNA against CHOP (Santa Cruz Biotechnology, Inc, sc-156,118), p38 (Santa Cruz Biotechnology, Inc, sc-29,433), ERK (RiboBio, siB0961281826-1-5) and non-targeting negative control siRNA (nc-siRNA) duplex, following the manufacturer's protocol. After overnight post-transfection, culture medium was replaced with fresh medium for an additional 24h. Then, cells were treated with TN and the expression of targeted protein was tested by Western blot.

\section{Statistical Analysis}

Results were expressed as the mean \pm standard deviation (SD). Comparisons between two groups were performed using the Student's $t$-test and between multiple groups using LSD-t analysis of variance. The difference was considered statistically significant if $P<0.05$. All analyses were performed using SPSS 23.0 software.

\section{Results \\ TN Suppressed Growth in Osteosarcoma Cells}

To investigate the cytotoxicity of TN against osteosarcoma, MTT assay was carried out in MG63 and U-2OS cells that were incubated with $5,10,25,50,100,250$ and $1000 \mathrm{nM}$ TN for $48 \mathrm{~h}$. We found that TN inhibited proliferation of MG63 and U-2OS cells in a dose-dependent manner. The $\mathrm{IC}_{50}$ for MG63 and U-2OS was 108.6 and 97.2, respectively (Figure 1A and B).

\section{TN Induced Apoptosis in Osteosarcoma Cells}

To further characterize the mode of cytotoxicity induced by $\mathrm{TN}$, we performed fluorescence cytochemical studies using flow cytometer. As shown in Figure 2 TN treatment $(10,50,100$, and $250 \mathrm{nM})$ induce apoptosis in a dosedependent manner in MG63 (Figure 2A and C) and U-2OS (Figure 2B and D) as evidenced by the significantly increased population of early and late apoptotic cells. 
These results suggest that the growth inhibitory potential of TN could be induction of apoptotic cell death.

\section{TN Induced an Increase of ROS Production in Osteosarcoma Cells}

Next, we examined whether the pro-apoptotic effect of TN on osteosarcoma cells involved increasing ROS levels, as it does in other cancer type. ${ }^{22}$ Interestingly, after treated with $10,25,50$ and $100 \mathrm{nM}$ for $48 \mathrm{~h}$, the ROS production increased significantly, and was reversed by $500 \mu \mathrm{M} \mathrm{NAC}$ pretreatment both in MG63 and U-2OS (Figure 3A and B). To further confirm whether ROS production resulted in cells apoptosis, Western blot analysis was carried to investigate the apoptotic markers. Decreased expression of Bcl-2 and Bcl-xL and increased expression of cleaved-caspase 3 and Bax indicated the onset of apoptosis in TN-treated cells treatment. However, treatment of cells with both TN and NAC exhibited a reversed effect substantiating that importance of ROS generation in apoptotic induction (Figure $3 \mathrm{C}$ and $\mathrm{D}$, Supplementary Figure S1). From the above findings, TN at a dose of $100 \mathrm{nM}$ was chosen for the subsequent assays. MTT assay and flow cytometry results shown that NAC pretreatment decreased growth inhibition and the population of apoptotic cells induced by TN (Figure 3E-J).

\section{TN Induced ER Stress-Apoptosis in Osteosarcoma Cells}

As increased ROS generation could cause ER stress, ${ }^{23}$ we investigated the expression of proteins associated with UPR. Our data have shown that treatment of cells with TN, increased the expression of PERK, p-eIF2, GRP78, ATF4 and CHOP (Figure 4A and B). Interestingly, the upregulation of PERK, p-eIF2, GRP78, ATF4 and CHOP was attenuated by NAC pretreatment (Figure 4A and B, Supplementary Figure S2). These findings substantiate that TN induced ER stress response in osteosarcoma cells. In fact, treatment of cells with $\mathrm{TN}$ in the presence of azoramide (Azo), a pharmacological inhibitor of ER stress response is shown to decrease growth inhibition and the population of apoptotic cells (Figure 4C-H). MTT assay and flow cytometry results shown that a decrease of growth inhibition and the population of apoptotic cells in TN-treated MG63 cells transfected with si-CHOP as compared to MG63 cells with TN treatment alone (Figure 4I-K). Again, Western blot analyses revealed a significant reduction in the expression cleaved-caspase 3 and Bax with an inverse relationship in the expression of Bcl-2 and Bcl-xL in TN-treated MG63 cells transfected with si-CHOP as compared to MG63 cells with TN treatment alone (Figure 4L, Supplementary Figure S3).

\section{TN Activated the P38 and ERK MAPK Signaling Pathways}

MAPK signaling plays an important role in cell proliferation and differentiation and dysregulation of this pathway has been reported in multiple cancer including osteosarcoma. ${ }^{24}$ Increased expression of phosphorylated p38 (p-p38) and ERK (p-ERK) as compared to p38 and ERK in TN-treated cells in addition to the decreased expression of p-JNK levels as shown in Figure 5 clearly demonstrates the efficiency of $\mathrm{TN}$ in the activation of ERK-MAPK pathway.

\section{TN Induced Cell ER Stress-Apoptosis via P38 and ERK MAPK Signaling Pathways}

In order to substantiate the role of MAPK in the induction of cell apoptosis, ${ }^{25}$ we further treated the cells with TN alone and TN combined with MAPK pharmacological inhibitors (including ERK inhibitor PD98059, p38 inhibitor SB203580 and JNK inhibitor SP600125). We found that PD98059 as well as SB203580 but not SP600125 pretreatment significantly decreased the growth inhibition, the population of apoptotic cells (Figure 6A-F) and the expression of PERK, p-eIF2, GRP78, ATF4 in MG63 and U-2OS (Figure 6G and $\mathrm{H}$, Supplementary Figure S4). Again, si-p38 and siERK were used to further confirm the above results. Obviously, si-p38 and si-ERK cells treated with TN showed a significant reduction in the growth inhibition, the population of apoptotic cells (Figure 6I-K) and the expression of PERK, p-eIF2, GRP78, ATF4 and CHOP were also reduced as compared to non-targeting siRNA in MG63 (Figure 6L, Supplementary Figure S5). These results demonstrated that TN induced cell apoptosis via p38 and ERK but not JNK MAPK signaling pathways.

\section{Discussion}

Triptonide is a key bioactive small molecule identified in a traditional Chinese medicine named Tripterygium wilfordii has a similar molecule structure with triptolide. ${ }^{26,27}$ Triptonide is structurally similar to triptolide except at C14 which is a carbonyl group in triptonide and a hydroxyl group in triptolide. ${ }^{28,29} \mathrm{~A}$ research report has shown that triptonide exhibited a varying degree of cytotoxicity which means the $\mathrm{IC}_{50}$ values of triptonide were higher than triptolide in BGC823 and MCF-7 cell lines, but was lower in Hela, KB and HL60 cell lines. ${ }^{30}$ Studies 
A

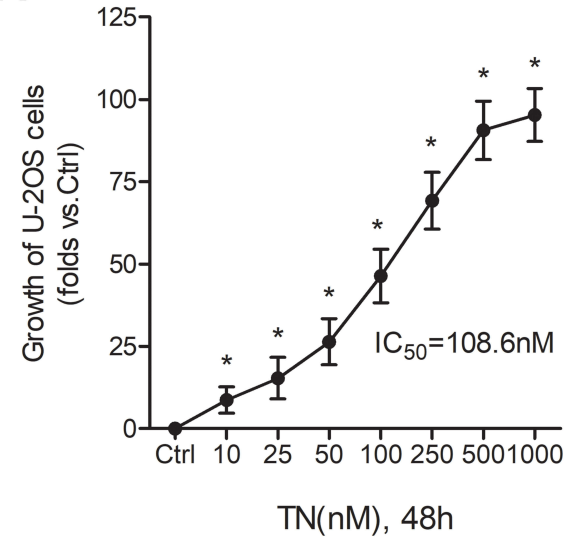

B

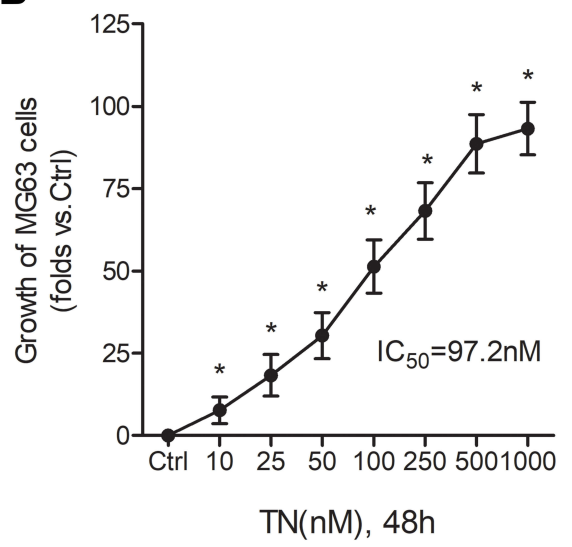

Figure I TN inhibits osteosarcoma cell growth. (A and B) Effect of TN on the viability of human osteosarcoma cells (MG63 and U-2OS cells). Cells were treated with various concentrations of TN for $48 \mathrm{~h}$ and viability was measured by MTT assay. The inhibition rate and IC 50 values are presented. The statistical difference $* P<0.05$ compared to the control group.
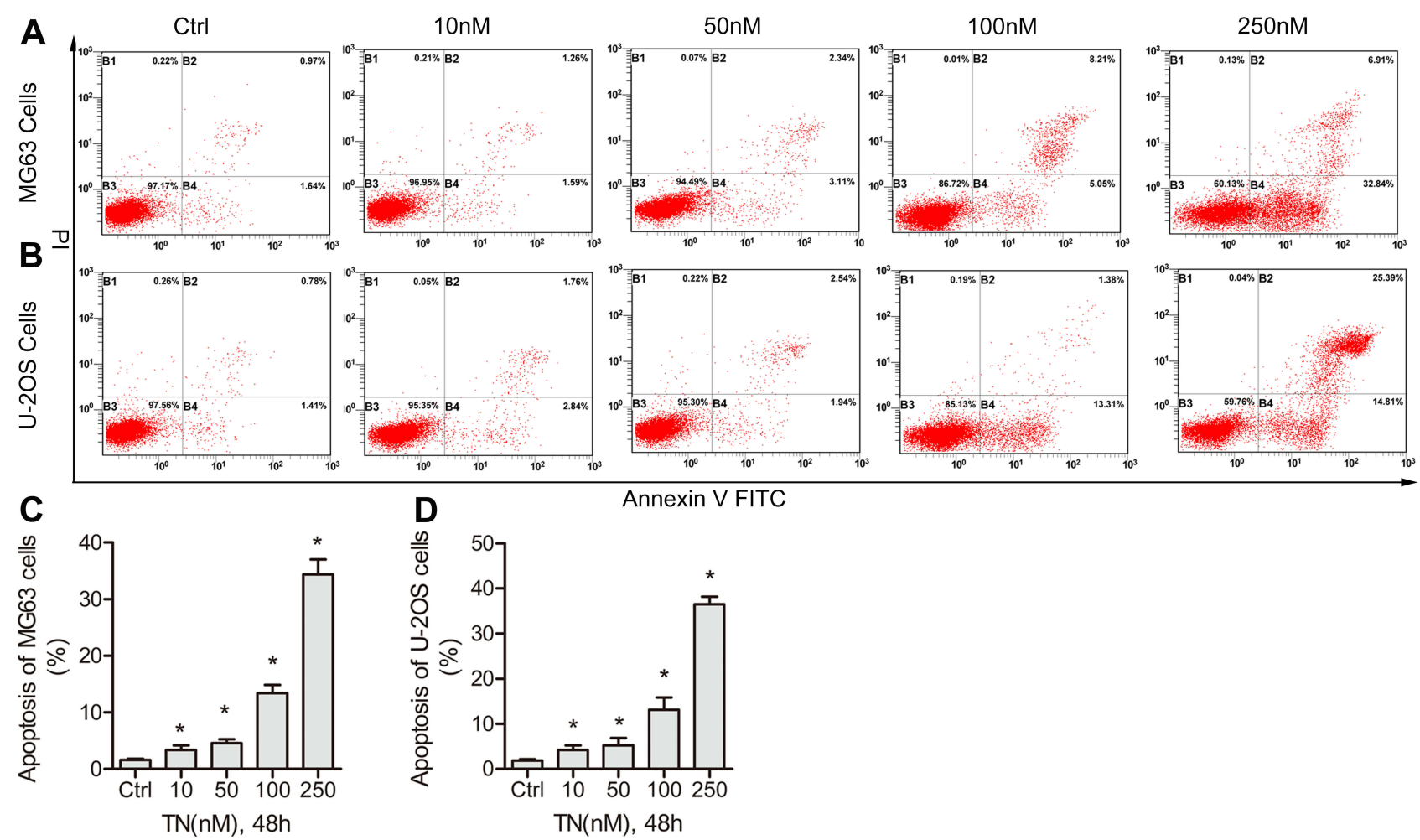

Figure 2 TN induces dose-dependent apoptosis in osteosarcoma cells. (A and B) The human osteosarcoma cells (MG63 and U-2OS cells) were treated with various doses of TN 10, 25, 50 and $100 \mathrm{nM}$ for 48h. (A and B) Flow cytometric analysis shows early and late apoptosis induced by TN in osteosarcoma cells (MG63 and U-2OS cells). (C and D) Histogram data indicate the percentage of apoptotic cells from three independent experiments. The statistical difference $* P<0.05$ compared with control group.

suggest that $\mathrm{TN}$ acts as an anti-tumor agent in various solid cancer. Wang et $\mathrm{al}^{22}$ indicate that $\mathrm{TN}$ inhibits human nasopharyngeal carcinoma cell growth via disrupting Lnc-RNA THOR-IGF2BP1 signaling. Zhang et $\mathrm{al}^{11}$ imply that TN inhibits lung cancer cell tumorigenicity by selectively attenuating the Shh-Glil signaling pathway.
Xiang et $\mathrm{al}^{31}$ prove that $\mathrm{TN}$ effectively suppresses gastric tumor growth and metastasis through inhibition of the oncogenic Notch1 and NF- $\kappa$ B signaling pathways. In this study, we attempted to explore the growth inhibitory effect of triptonide in osteosarcoma cells and found that triptonide at nano-scale concentration dramatically inhibited the 

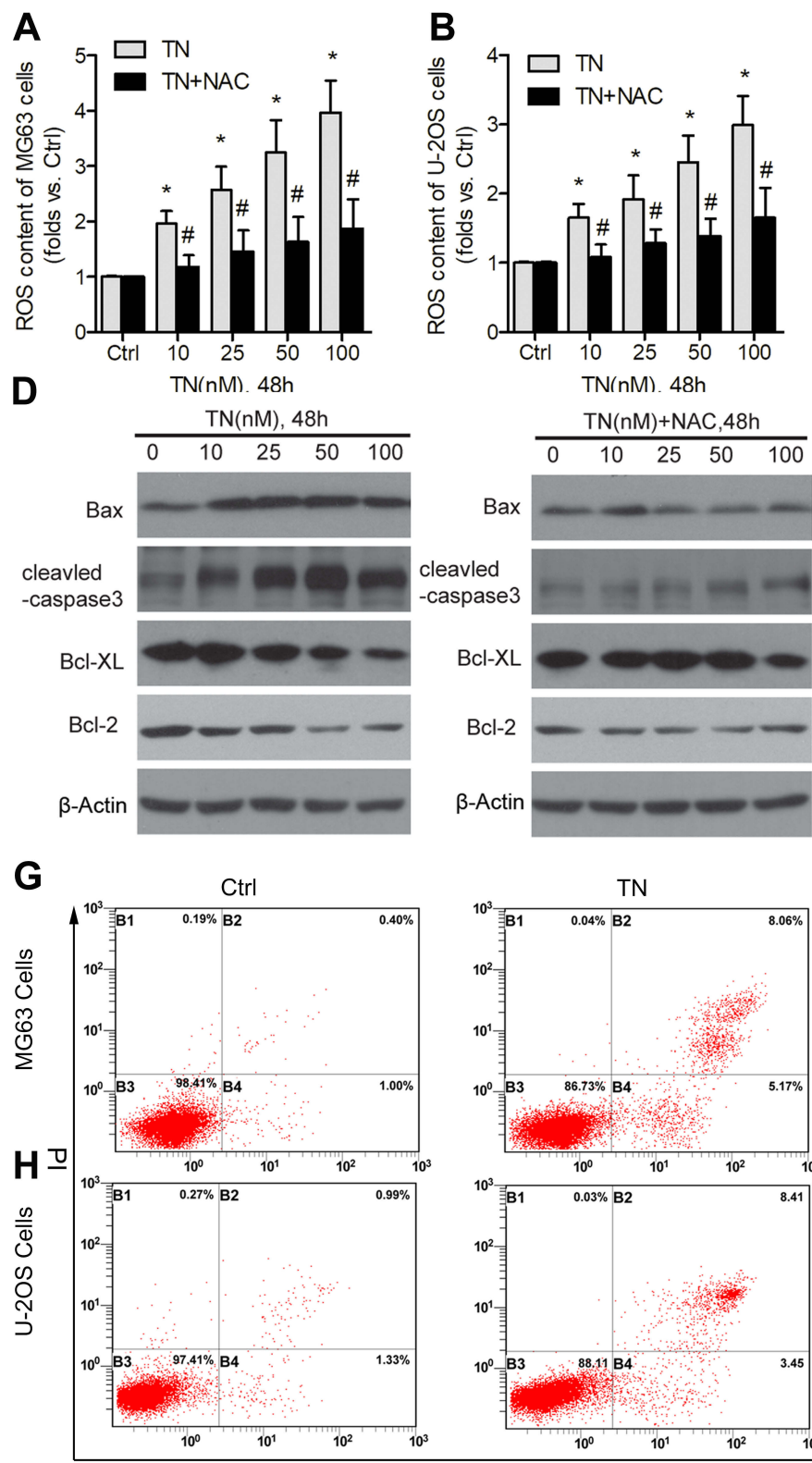
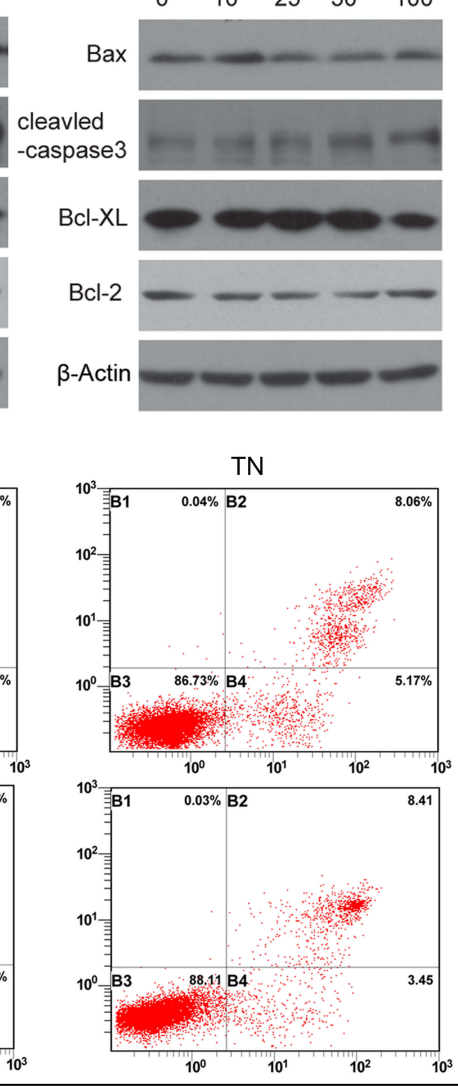
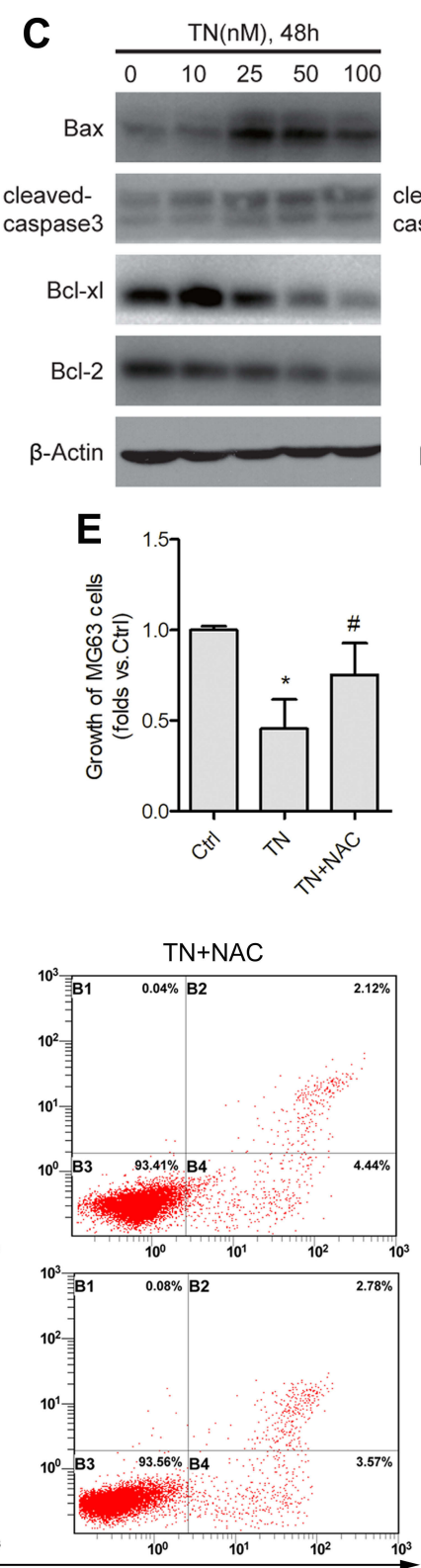

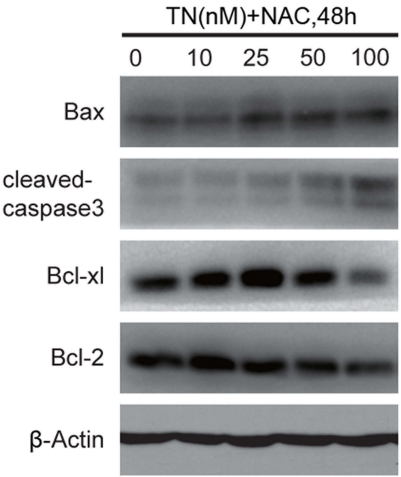

$\mathbf{F}$
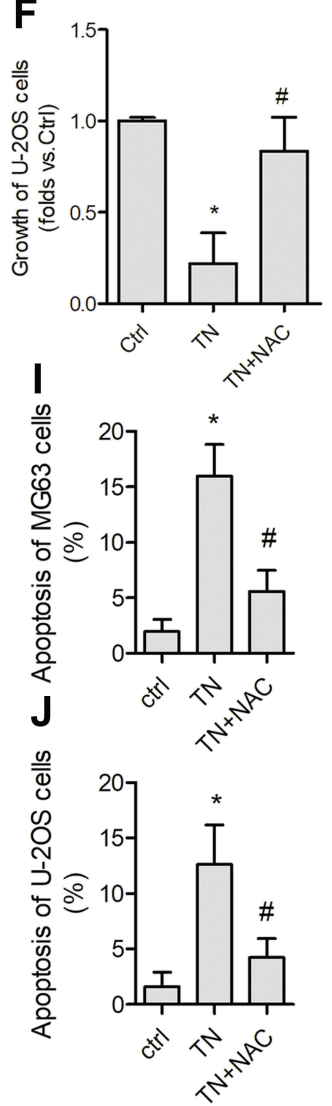

Figure 3 TN increases intracellular ROS production and induces apoptosis in osteosarcoma cells. (A and B) Cells were treated with various concentration of TN $10,25,50$ and $100 \mathrm{nM}$ alone or co-treated with NAC $(500 \mu \mathrm{M})$ for $48 \mathrm{~h}$. The Intracellular ROS levels were measured by DCFH-DA (I0 $\mu$ M) in both MG63 and U-2OS cells. The statistical difference ${ }^{* P}<0.05$ or ${ }^{\#} P<0.05$ compared with control group. (C and D) Western blot analysis shows the expression level of anti- and pro-apoptotic protein Bax, cleaved caspase-3, Bcl-XL and $\mathrm{Bcl}-2$ upon TN alone or co-treatment with NAC for $48 \mathrm{~h}$. $\beta$-Actin used as internal control. Data represent similar results from three independent experiments. (E and F) MTT assay shows the ROS-dependent cytotoxic effect of TN. (G-J) The flow cytometric analysis of ROS-dependent apoptosis induced by TN in both MG63 and U-2OS cells. The statistical difference $* P<0.05$ compared with control group or ${ }^{\#} P<0.05$ compared with TN-alone-treated group.

growth in MG63 and U-2OS and also induced apoptosis in a dose-dependent manner. Western blot results indicated that TN effectively altered Bax/Bcl-2 ratio favoring apoptosis induction in U-2OS as evidenced by the increased expression of pro-apoptosis proteins Bax and cleavedcaspase 3 and decreased expression of anti-apoptosis protein $\mathrm{Bcl}-2$ and $\mathrm{Bcl}-\mathrm{XL}$.
Various anticancer agents have been shown to promote ROS generation in cancer cells. ${ }^{32,33}$ Study by Rajamanickam et $\mathrm{al}^{20}$ indicates that allylated curcumin analog CA6 directly binds to and inhibiting the activity of TrxR1 resulting in the increase of ROS. Others suggest that Chinese chemical like ginsenoside Rh4, Luteolin may disturb the mitochondria function to promote the 
A

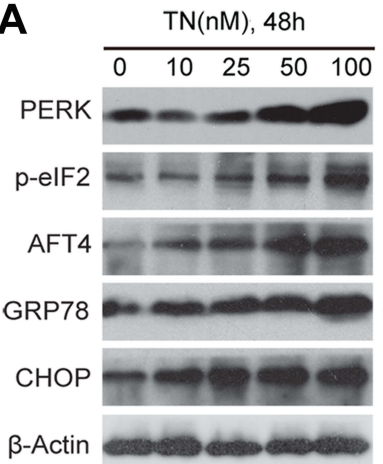

C
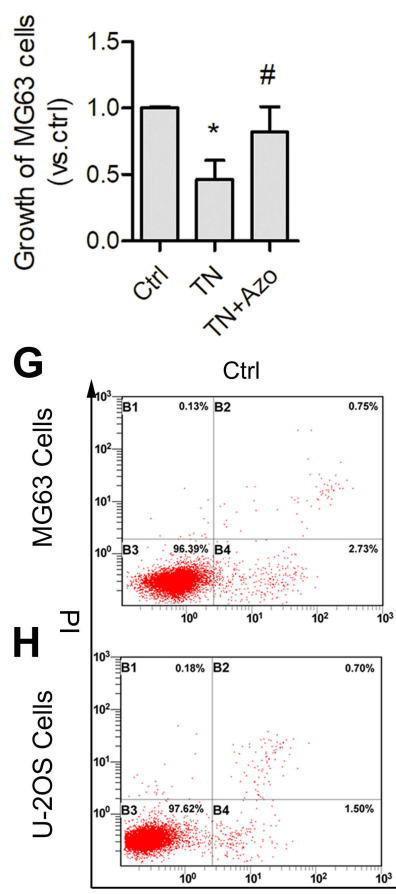

K

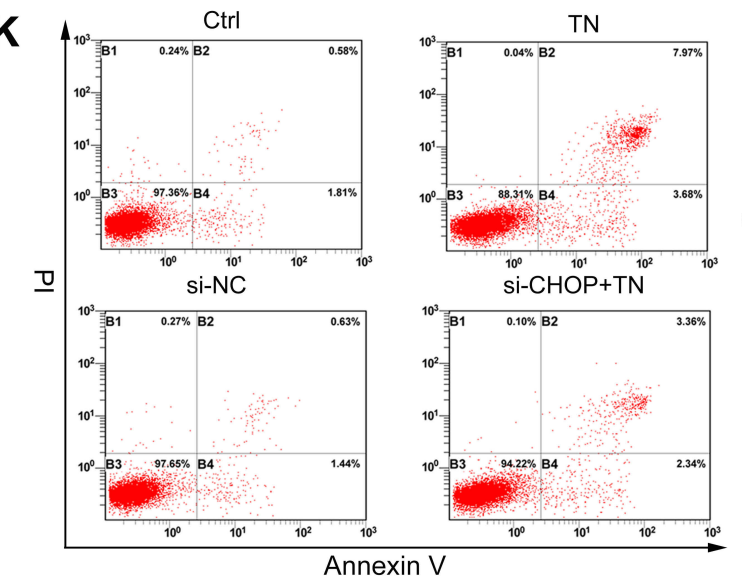

\begin{tabular}{lllll}
\hline 0 & 10 & 25 & 50 & 100
\end{tabular}

PERK

p-elF2

AFT4

GRP78

CHOP

$\beta$-Actin

D
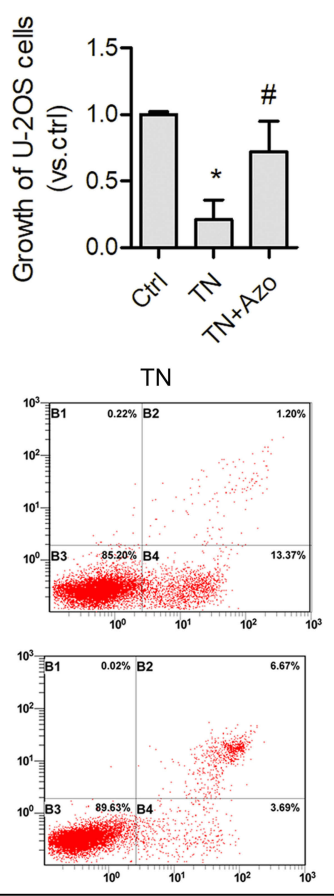

Annexin V

$\mathbf{L}$

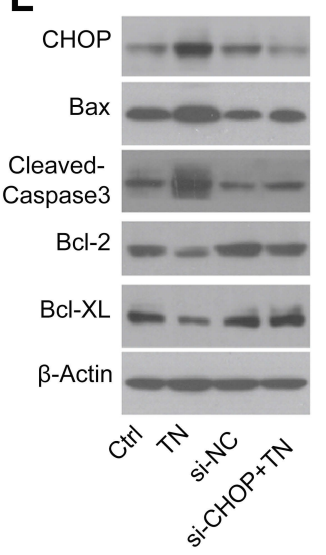

B
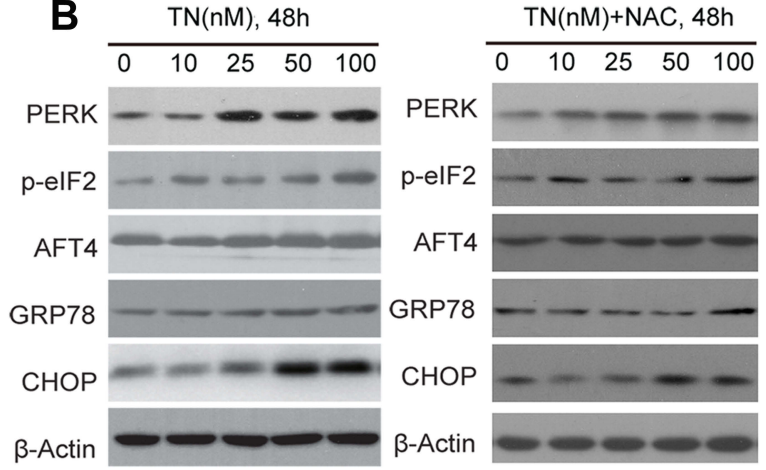

E

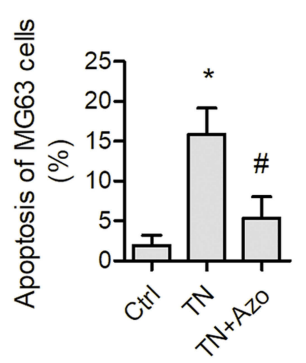

F
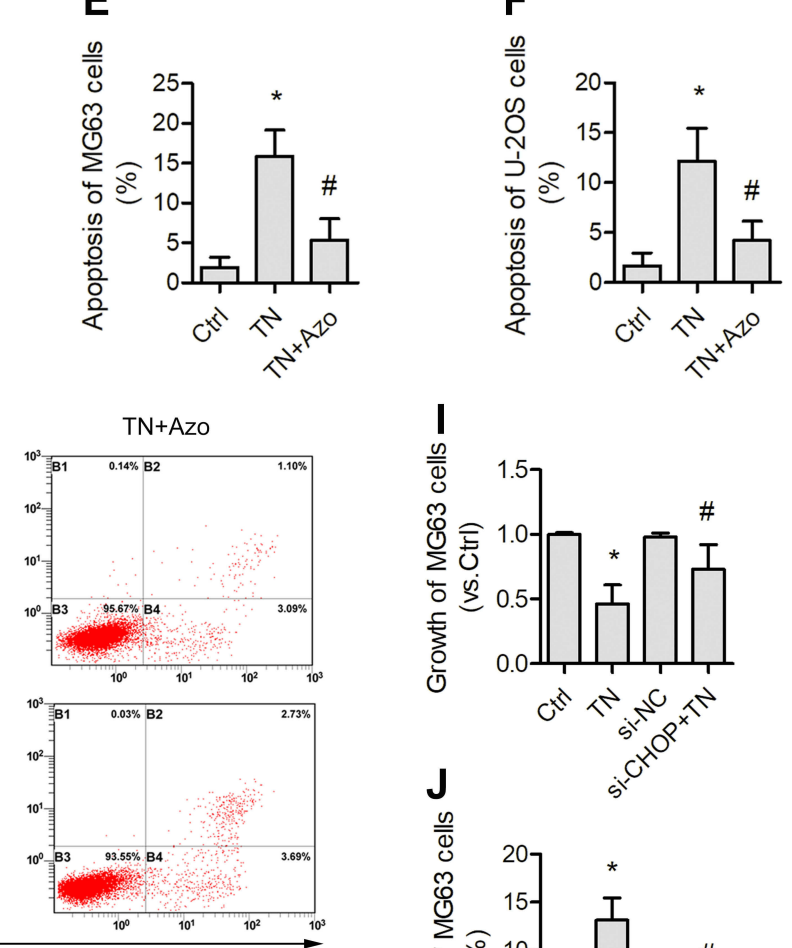

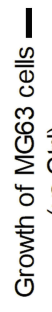
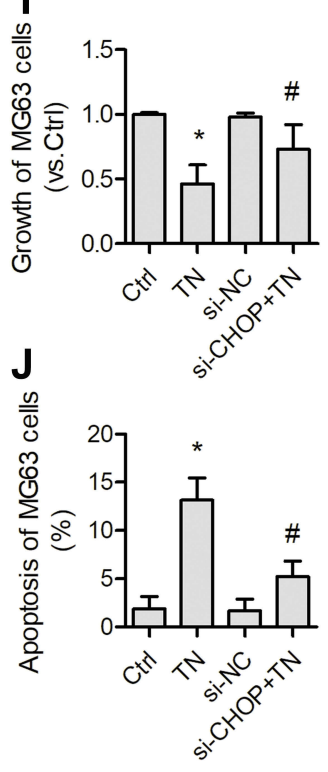

Figure 4 TN induces ROS-dependent ER stress and apoptotic cell death in osteosarcoma cells. (A and B) Western blot analysis shows the expression level of ER stressrelated proteins PERK, p-elF2, GRP78, ATF4 and CHOP in both MG63 and U-2OS cells upon treatment with TN alone or co-treated with NAC (500 $\mu$ M) for 48h. (C and D) MTT assay shows the cytotoxic effect of TN upon co-treatment with ER stress inhibitor azoramide (Azo). (E-H) Apoptosis induced by TN upon co-treatment with azoramide. MG63 cells transfected with CHOP siRNA and then were exposed to TN for $48 \mathrm{~h}$. (I-K) Cell viability and apoptotic cells were determined by MTT assay or Annexin V/PI staining. (L) MG63 cells were transfected with CHPO siRNA and expression of ER stress and apoptosis proteins were determined by Western blotting. $\beta$-Actin used as internal control. Data represent similar results from three independent experiments. The statistical difference $* P<0.05$ compared with control group or ${ }^{\#} P<0.05$ compared with $\mathrm{TN}$-alone-treated group. 
production of ROS. ${ }^{34,35}$ In line with these findings, the present study also revealed the increased ROS accumulation in MG63 and U-2OS cells treated with TN which was shown to be reversed by the ROS scavenger NAC. However, it needs to further identify the underline mechanism. Another interesting finding of the current study is that TN failed to induce apoptosis by NAC pretreatment as evidenced by the annexin $\mathrm{V}$ assay, which in turn substantiate that TN induced ROS-mediated apoptosis in osteosarcoma cells.

Oxidative stress as a result of ROS imbalance has been shown to induce ER stress and as a consequence of ER an adaptive mechanism named unfolded protein response is activated in order to bring ER homeostasis. ${ }^{36,37}$ Glucose regulated protein 78 (GRP78) a chief sensor of ER stress activates UPR resulting in upregulation of PERK following phosphorylating eukaryotic initiation factor-2 $\alpha$ (eIF2 $\alpha$ ) and increasing translation of ATF4. With the upregulation of chaperone proteins such as GRP78, ATF4 increased the pro-apoptotic transcriptional regulator $\mathrm{CHOP}^{38}$ As a transcription factor CHOP plays a key role in ER stressmediated apoptosis and has been suggested as a marker of ER stress-induced apoptosis. ${ }^{38}$ Upregulation of PERK, p-eIF2, GRP78, ATF4 and CHOP in TN-treated cells clearly indicated that $\mathrm{TN}$ elicits oxidative driven ER stress-mediated apoptosis. These findings were further confirmed when si-CHOP and ER stress response pharmacological inhibitors azoramide abolish apoptotic effect of TN.

Mitogen-activated protein kinases (MAPKs) are a complex interconnected signaling cascade that includes ERK, p38, and JNK MAPK subfamilies, which are crucial regulators of cellular physiology and their frequent involvement in oncogenesis, tumor progression, and drug resistance has also been widely reported and these members of MAPK cascade remain an important target for anti-tumor agents. ${ }^{24}$ Studies have also shown that activation of MAPK signaling pathways result in cell apoptosis. ${ }^{25}$ An existence of cross talk has also been demonstrated between MAPK signaling and ER stress ${ }^{23,39}$ and activation of MAPK could result in up-regulation of CHOP and cell apoptosis. ${ }^{40}$ Herein we reported that TN-treated cells showed an increased phosphorylation of ERK and p38, but not JNK, reflecting the activation of ERK and p38 MAKP pathway. Further we found that the p38 inhibitor SB203580 and ERK inhibitor PD98059 but not the JNK inhibitor SP600125 decreased the expression of PERK, p-EIF2, GRP78, ATF4 and CHOP which is accompanied by decreased in apoptotic cells with SB203580 and PD98059 but not with SP600125 pretreatment. Knock-down of p38 and ERK by transfecting si-p38
A

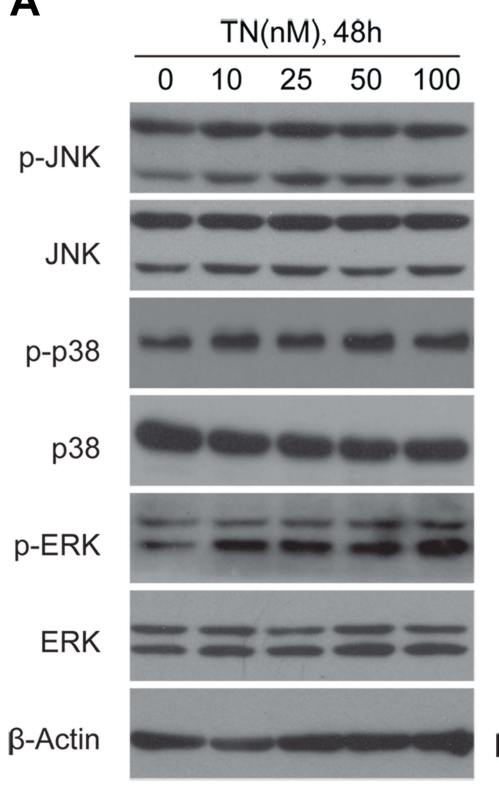

B

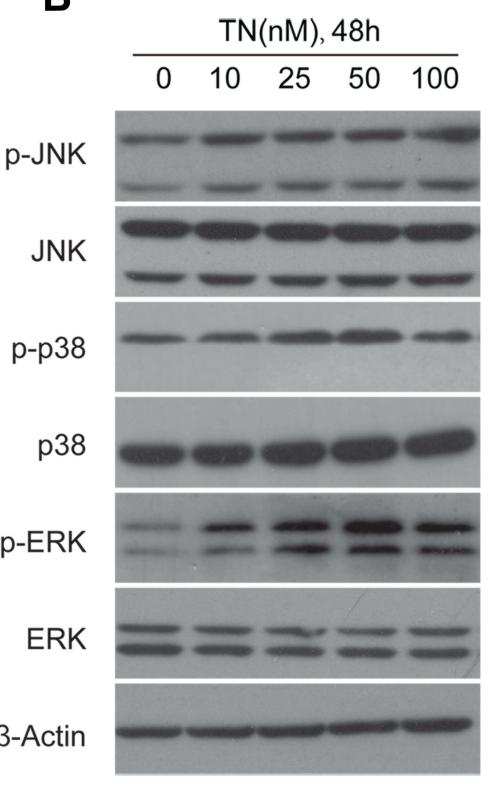

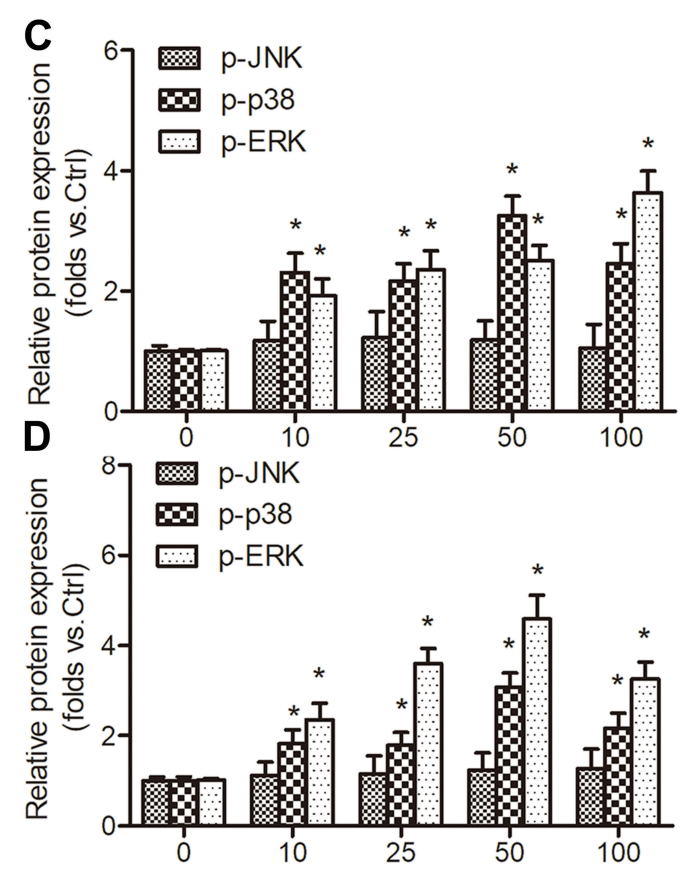

Figure 5 TN dose-dependently activates $\mathrm{p} 38$ and ERK MAPK signaling pathway in osteosarcoma cells. (A and B) MG63 and U-2OS cells were treated with various concentration of 10, 25, 50 and $100 \mathrm{nM}$ for 48 h. Western blot analysis shows protein levels of $\mathrm{p}$-JNK, $\mathrm{p}$-p38 and p-ERK. (C and D) The relative protein expression of $\mathrm{p}$-JNK, $\mathrm{P}$-p38 and $\mathrm{p}$-ERK were normalized by control group. Data represent similar results from three independent experiments. JNK, $\mathrm{P} 38$, ERK and $\beta$-Actin were used as internal control. The statistical difference $* P<0.05$ compared with control group. 

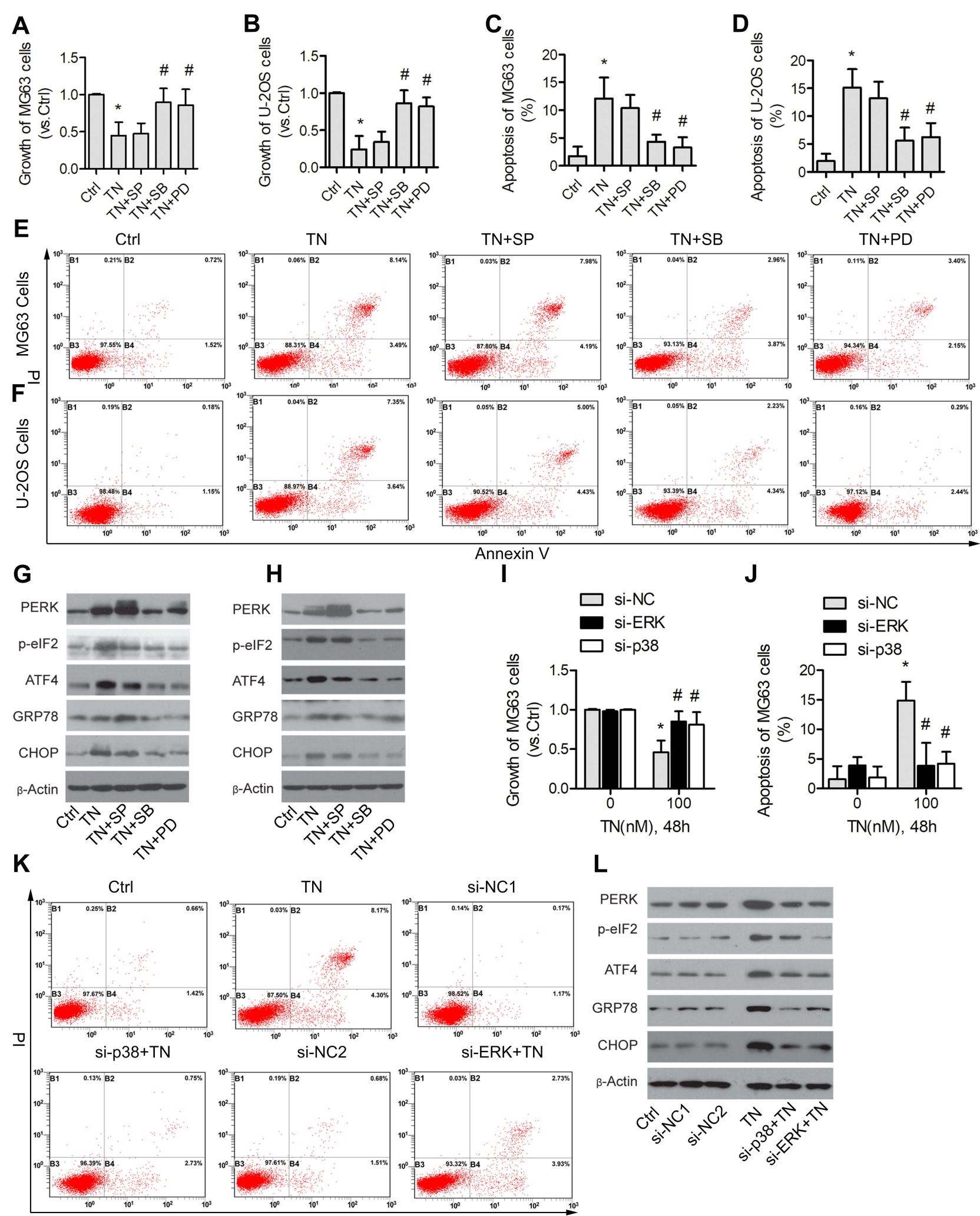

L

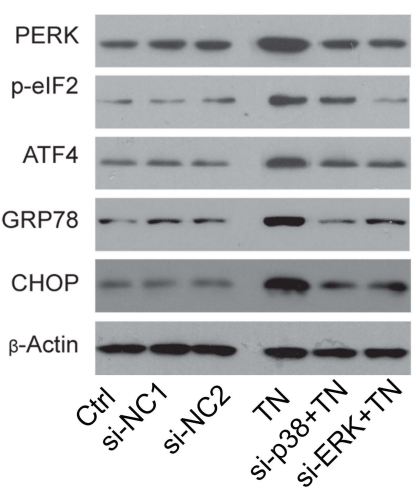

Figure 6 TN induces cell ER stress-mediated apoptosis via p38 and ERK MAPK signaling pathways. MG63 and U-2OS cells were treated with TN alone (I00nM) or cotreated with PD98059, SB203580 and SP600I 25 for 48h. (A-F) Cell viability and apoptotic cells were determined by MTT assay or Annexin V/PI staining. (G and H) Protein levels of PERK, p-elF2, GRP78, ATF4 and CHOP were detected by Western blotting. MG63 cells transfected with siRNA against p38 or ERK and then exposed to TN. (I-K) Cell viability and apoptotic cells were determined by MTT assay or Annexin V/PI staining. (L) Protein levels of PERK, P-elF2, GRP78, ATF4 and CHOP were detected by Western blotting. $\beta$-Actin were used as internal control. Data represent similar results from three independent experiments. The statistical difference $* P<0.05$ compared with control group or ${ }^{\#} P<0.05$ compared with TN-alone-treated group. 
and si-ERK plasmid also shown the similar results as cells pre-treated with SB203580 and PD98059. These data indicated that TN induces p38 and ERK MAPK signaling pathways to promote ER stress induced-apoptosis.

In summary, the outcome of the present study clearly depicts that $\mathrm{TN}$ treatment causes excess intracellular ROS accumulation and inhibits the growth of OS cells. The excess ROS production and oxidative stress cause ER stress-mediated apoptosis via induction of p38 and ERK MAPK signaling pathway. TN acts as a potential antitumor agent which could be considered for further investigations to implement in clinical use in near future.

\section{Acknowledgments}

The study was supported by the National Natural Science Foundation of China (grant number 81803778), Science and Technology Department of Lishui City (grant number 2016GYX39) and Zhejiang Province Medical and Health Care Project (grant number 2017KY170).

\section{Disclosure}

The authors report no conflicts of interest in this work.

\section{References}

1. Stiller CA. International patterns of cancer incidence in adolescents. Cancer Treat Rev. 2007;33(7):631-645. doi:10.1016/j. ctrv.2007.01.001

2. Harrison DJ, Geller DS, Gill JD, et al. Current and future therapeutic approaches for osteosarcoma. Expert Rev Anticancer Ther. 2018;18 (1):39-50. doi:10.1080/14737140.2018.1413939

3. Hattinger CM, Fanelli M, Tavanti E, et al. Doxorubicin-resistant osteosarcoma: novel therapeutic approaches in sight? Future Oncol. 2017;13(8):673-677. doi:10.2217/fon-2016-0519

4. Yang J, Zhang W. New molecular insights into osteosarcoma targeted therapy. Curr Opin Oncol. 2013;25(4):398-406. doi:10.1097/ CCO.0b013e3283622c1b

5. Marcus DM. Comparison of Tripterygium wilfordii Hook $F$ with methotrexate in the treatment of rheumatoid arthritis. Ann Rheum Dis. 2014;73(9):e56. doi:10.1136/annrheumdis-2014-205971

6. Wang Q, Meng J, Dong A, et al. The pharmacological effects and mechanism of Tripterygium wilfordii Hook $\mathrm{F}$ in central nervous system autoimmunity. J Altern Complement Med. 2016;22(7):496-502. doi:10.1089/acm.2016.0004

7. Li -X-X, Du F-Y, Liu H-X, et al. Investigation of the active components in Tripterygium wilfordii leading to its acute hepatotoxicity and nephrotoxicity. J Ethnopharmacol. 2015;162:238-243. doi:10.1016/j. jep.2015.01.004

8. Tao X, Lipsky PE. The Chinese anti-inflammatory and immunosuppressive herbal remedy Tripterygium wilfordii Hook F. Rheum Dis Clin North Am. 2000;26(1):29-50. doi:10.1016/S0889-857X(05) 70118-6

9. Wong KF, Yuan Y, Luk JM. Tripterygium wilfordii bioactive compounds as anticancer and anti-inflammatory agents. Clin Exp Pharmacol Physiol. 2012;39(3):311-320. doi:10.1111/j.14401681.2011.05586.x
10. Han H, Du L, Cao Z, et al. Triptonide potently suppresses pancreatic cancer cell-mediated vasculogenic mimicry by inhibiting expression of VE-cadherin and chemokine ligand 2 genes. Eur J Pharmacol. 2018;818:593-603. doi:10.1016/j.ejphar.2017.11.019

11. Zhang M, Tan S, Yu D, et al. Triptonide inhibits lung cancer cell tumorigenicity by selectively attenuating the Shh-Glil signaling pathway. Toxicol Appl Pharmacol. 2019;365:1-8. doi:10.1016/j. taap.2019.01.002

12. Yang P, Dong F, Zhou Q. Triptonide acts as a novel potent anti-lymphoma agent with low toxicity mainly through inhibition of proto-oncogene Lyn transcription and suppression of Lyn signal pathway. Toxicol Lett. 2017;278:9-17. doi:10.1016/j.toxlet.2017.06.010

13. Sisinni L, Pietrafesa M, Lepore S, et al. Endoplasmic reticulum stress and unfolded protein response in breast cancer: the balance between apoptosis and autophagy and its role in drug resistance. Int J Mol Sci. 2019;20(4):857. doi:10.3390/ijms20040857

14. Wang $\mathrm{Z}$, Yu K, Hu Y, et al. Schisantherin A induces cell apoptosis through ROS/JNK signaling pathway in human gastric cancer cells. Biochem Pharmacol. 2020;173:113673. doi:10.1016/j.bcp.2019.113673

15. Yang Y, Liu PY, Bao W, et al. Hydrogen inhibits endometrial cancer growth via a ROS/NLRP3/caspase-1/GSDMD-mediated pyroptotic pathway. BMC Cancer. 2020;20(1):28. doi:10.1186/s12885-0196491-6

16. Sun X-L, Zhang X-W, Zhai H-J, et al. Magnoflorine inhibits human gastric cancer progression by inducing autophagy, apoptosis and cell cycle arrest by JNK activation regulated by ROS. Biomed Pharmacother. 2020;125:109118. doi:10.1016/j.biopha.2019.109118

17. Dauer P, Sharma NS, Gupta VK, et al. ER stress sensor, glucose regulatory protein 78 (GRP78) regulates redox status in pancreatic cancer thereby maintaining "stemness". Cell Death Dis. 2019;10 (2):132. doi:10.1038/s41419-019-1408-5

18. Yang KM, Kim BM, Park JB. omega-Hydroxyundec-9-enoic acid induces apoptosis through ROS-mediated endoplasmic reticulum stress in non-small cell lung cancer cells. Biochem Biophys Res Commun. 2014;448(3):267-273. doi:10.1016/j.bbrc.2014.04.111

19. Wu J, Zheng W, Rong L, et al. Bicyclol exerts an anti-tumor effect via ROS-mediated endoplasmic reticulum stress in human renal cell carcinoma cells. Biomed Pharmacother. 2017;91:1184-1192. doi:10.1016/j.biopha.2017.05.041

20. Rajamanickam V, Yan T, Wu L, et al. Allylated curcumin analog CA6 inhibits TrxR1 and leads to ROS-dependent apoptotic cell death in gastric cancer through Akt-FoxO3a. Cancer Manag Res. 2020;12:247. doi:10.2147/CMAR.S227415

21. Tvedennyborg P, Bergmann TK, Lykkesfeldt J. Basic \& clinical pharmacology \& toxicology policy for experimental and clinical studies. Basic Clin Pharmacol Toxicol. 2018;123:233-235. doi:10.1111/bcpt.13059

22. Wang -S-S, Lv Y, Xu X-C, et al. Triptonide inhibits human nasopharyngeal carcinoma cell growth via disrupting Lnc-RNA THOR-IGF2BP1 signaling. Cancer Lett. 2019;443:13-24. doi:10.1016/j.canlet.2018.11.028

23. Darling NJ, Cook SJ. The role of MAPK signalling pathways in the response to endoplasmic reticulum stress. Biochim Biophys Acta. 2014;1843(10):2150-2163. doi:10.1016/j.bbamcr.2014.01.009

24. Sun Y, Liu W-Z, Liu T, et al. Signaling pathway of MAPK/ERK in cell proliferation, differentiation, migration, senescence and apoptosis. J Recept Signal Transduct Res. 2015;35(6):600-604. doi:10.3109/10799893.2015.1030412

25. Sui X, Kong N, Ye L, et al. p38 and JNK MAPK pathways control the balance of apoptosis and autophagy in response to chemotherapeutic agents. Cancer Lett. 2014;344(2):174-179. doi:10.1016/j. canlet.2013.11.019

26. Tengchaisri T, Chawengkirttikul R, Rachaphaew N, et al. Antitumor activity of triptolide against cholangiocarcinoma growth in vitro and in hamsters. Cancer Lett. 1998;133(2):169-175. doi:10.1016/S0304$3835(98) 00222-5$ 
27. Phillips PA, Dudeja V, McCarroll JA, et al. Triptolide induces pancreatic cancer cell death via inhibition of heat shock protein 70 Cancer Res. 2007;67(19):9407-9416. doi:10.1158/0008-5472.CAN07-1077

28. He L, Liang Z, Zhao F, et al. Modulation of IL-37 expression by triptolide and triptonide in THP-1 cells. Cell Mol Immunol. 2015;12 (4):515. doi:10.1038/cmi.2014.92

29. Wong K-F, Chan JK, Chan K-L, et al. Immunochemical characterization of the functional constituents of Tripterygium wilfordii contributing to its anti-inflammatory property. Clin Exp Pharmacol Physiol. 2008;35(1):55-59. doi:10.1111/j.1440-1681.2007.04740.x

30. Ning L, Qu G, Ye M, et al. Cytotoxic biotransformed products from triptonide by Aspergillus niger. Planta Med. 2003;69(9):804-808.

31. Xiang S, Zhao Z, Zhang T, et al. Triptonide effectively suppresses gastric tumor growth and metastasis through inhibition of the onco-

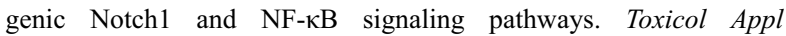
Pharmacol. 2020;388:114870. doi:10.1016/j.taap.2019.114870

32. Wasim L, Chopra M. Synergistic anticancer effect of panobinostat and topoisomerase inhibitors through ROS generation and intrinsic apoptotic pathway induction in cervical cancer cells. Cell Oncol (Dordr). 2018;41(2):201-212. doi:10.1007/s13402-017-0366-0

33. Tang JY, Ou-Yang F, Hou MF, et al. Oxidative stress-modulating drugs have preferential anticancer effects - involving the regulation of apoptosis, DNA damage, endoplasmic reticulum stress, autophagy, metabolism, and migration. Semin Cancer Biol. 2018.
34. Wang Q, Wang H, Jia Y, et al. Luteolin induces apoptosis by ROS/ER stress and mitochondrial dysfunction in glioblastoma. Cancer Chemother Pharmacol. 2017;79(5):1031-1041. doi:10.1007/s00280017-3299-4

35. Wu Q, Deng J, Fan D, et al. Ginsenoside Rh4 induces apoptosis and autophagic cell death through activation of the ROS/JNK/p53 pathway in colorectal cancer cells. Biochem Pharmacol. 2018;148:64-74. doi:10.1016/j.bcp.2017.12.004

36. Moloney JN, Cotter TG. ROS signalling in the biology of cancer. Semin Cell Dev Biol. 2018;80:50-64. doi:10.1016/j.semcdb.2017.05.023

37. Zhang Z, Zhang L, Zhou L, et al. Redox signaling and unfolded protein response coordinate cell fate decisions under ER stress. Redox Biol. 2019;25:101047.

38. Li Y, Guo Y, Tang J, et al. New insights into the roles of CHOP-induced apoptosis in ER stress. Acta Biochim Biophys Sin (Shanghai). 2015;47(2):146-147. doi:10.1093/abbs/gmu128

39. Sreedhar R, Giridharan VV, Arumugam S, et al. Role of MAPK-mediated endoplasmic reticulum stress signaling in the heart during aging in senescence-accelerated prone mice. Biofactors. 2016;42(4):368-375. doi:10.1002/biof.1280

40. Chakraborty S, Ghosh S, Banerjee B, et al. Mephebrindole, a synthetic indole analog coordinates the crosstalk between p38MAPK and eIF2alpha/ATF4/CHOP signalling pathways for induction of apoptosis in human breast carcinoma cells. Apoptosis. 2016;21(10):1106-1124. doi:10.1007/s10495-016-1268-8

\section{Publish your work in this journal}

Cancer Management and Research is an international, peer-reviewed open access journal focusing on cancer research and the optimal use of preventative and integrated treatment interventions to achieve improved outcomes, enhanced survival and quality of life for the cancer patient.
The manuscript management system is completely online and includes a very quick and fair peer-review system, which is all easy to use. Visit http://www.dovepress.com/testimonials.php to read real quotes from published authors. 